\title{
Nucleosynthesis in Helium Shell Flashes on Accreting Carbon-Oxygen White Dwarfs
}

\author{
Yasuomi Kamiya* ${ }^{* \dagger}$ \\ Department of Astronomy, Graduate School of Science, The University of Tokyo, 7-3-1 Hongo, \\ Bunkyo-ku, Tokyo 113-0033, Japan \\ Kavli Institute for the Physics and Mathematics of the Universe, Todai Institutes for Advanced \\ Study, The University of Tokyo, 5-1-5 Kashiwanoha, Kashiwa, Chiba 277-8583, Japan \\ E-mail: vasuomi.kamiyadipmu.jp
}

\begin{abstract}
An early-time spectrum of a Type Ia supernova (SN Ia) gives us clues to understand chemical abundance in the outer layers of the progenitor white dwarf (WD) before the SN explosion or at the final stage of the explosive burning. There are some features in the early-time spectra of SNe Ia which contradict current theoretical models of an exploding WD. For example, the early-time spectra of some SNe Ia show absorption lines faster than the photospheric velocity (e.g., Si and $\mathrm{Ca}$ ), called high velocity features (HVFs). The interaction between the ejecta and circumstellar material has been suggested to result in the HVFs, but their origin has been poorly understood. The HVF elements in the outer layers of the SN ejecta might also be the origin. Nucleosynthesis in the He shell flashes before the SN explosion could help us to understand such features. We have calculated the progress and nucleosynthesis of the He shell flashes on the surface of the accreting $\mathrm{C}-\mathrm{O}$ WD with some approximations. The preliminary results suggest that a significant amount of the elements heavier than $\mathrm{C}$ and $\mathrm{O}$ (such as $\mathrm{Si}$ and $\mathrm{S}$ ) could be synthesized in the outer layers of the WD.
\end{abstract}

XII International Symposium on Nuclei in the Cosmos

August 5-12, 2012

Cairns, Australia

\footnotetext{
${ }^{*}$ Speaker.

${ }^{\dagger}$ Research Fellow of the Japan Society for the Promotion of Science.
} 


\section{Introduction}

A Type Ia supernova (SN Ia) is thought to be a thermonuclear explosion of a C-O white dwarf (WD) in a close binary system (e.g., [四-团]). The WD explodes when its mass $\left(M_{\mathrm{WD}}\right)$ becomes close to the Chandrasekhar mass $\left(M_{\mathrm{WD}}, \sim 1.4 M_{\odot}\right)$ by accreting hydrogen-rich matter from its companion star. Thus, $\mathrm{SNe}$ Ia are expected to have similar properties.

Early-time spectra of several SNe Ia, taken before the maximum brightness, show unusual absorption lines of $\mathrm{Ca}$ and $\mathrm{Si}$ (e.g., [可]). Their line velocity is faster than that of the photosphere, so that these high-velocity lines are referred to as high velocity features (HVFs). The origin of HVFs has not been understood clearly. It has been thought that the HVF could result from the interaction between the SN ejecta and circumstellar matter, but not fully confirmed yet.

Another possible explanation for the HVFs is the existence of the HVF elements in the outermost layer of the progenitor WD. However, the abundance in the outer layer of the accreting WD has not been studied well. As the progenitor WD increases its mass, the $\mathrm{H}$ accreted to the WD is burnt into $\mathrm{He}$ on the surface of the WD. This $\mathrm{He}$ is then burnt into $\mathrm{C}$ and $\mathrm{O}$ through He shell flashes. The abundance of the elements in the accreted layer is thus determined by the recurring flashes. Since it is difficult to calculate these so many flashes, the $\mathrm{C}$ and $\mathrm{O}$ are simply assumed to accrete with almost equal mass fractions after the flashes.

In this study, with some approximations, the progress and nucleosyntheis of He shell flashes on the WD with $M_{\mathrm{WD}} \sim M_{\mathrm{Ch}}$ are examined.

\section{Calculation}

We consider a WD which consists of $\mathrm{C}$ and $\mathrm{O}$ with the same mass fraction and has a $\mathrm{He}$ envelope on it. Given $M_{\mathrm{WD}}$ and the envelope mass $\left(M_{\mathrm{env}}\right)$, we can derive the structure of the envelope in hydrostatic equilibrium quasi-analytically $[6,0]$. Such quasi-analytic model is also applied in calculating the nucleosynthesis of $\mathrm{O}-\mathrm{Ne}-\mathrm{Mg}$ novae $[[]]$. The pressure and density at the base of the envelope are given by

$$
P_{\mathrm{b}}=\frac{G M_{\mathrm{WD}} M_{\mathrm{env}}}{4 \pi R_{\mathrm{WD}}^{4}} f_{\mathrm{b}} \text { and } \rho_{\mathrm{b}}=\frac{M_{\mathrm{env}}}{4 \pi R_{\mathrm{WD}}^{3}} V_{\mathrm{b}} f_{\mathrm{b}}
$$

where the subscript "b" denotes the value at the base of the envelope, and $R_{\mathrm{WD}}$ is the WD radius which is expressed as a function of $M_{\mathrm{WD}}{ }^{1}$. In the above equations, $V$ and $f$ are described by

$$
V \equiv-\frac{d \ln P}{d \ln r}=\frac{G M_{r} \rho}{r P} \text { and } f(x, N) \equiv \frac{x^{N+1}(1-x)^{3-N}}{(N+1) B_{x}(N+1,3-N)}(0<f<1) .
$$

Here, the polytropic index $N$ is assumed to be adiabatic and constant throughout the envelope, $x$ is defined as $x \equiv(N+1) / V(0<x<1)$, and $B_{x}$ denotes the incomplete beta function ${ }^{2}$. Assuming

\footnotetext{
${ }^{1}$ The relation between $R_{\mathrm{WD}}$ and $M_{\mathrm{WD}}$ is obtained by solving the structures of isothermal $\left(10^{7} \mathrm{~K}\right) \mathrm{C}-\mathrm{O}$ WDs with various central density.$$
{ }^{2} N /(N+1) \equiv d \ln \rho / d \ln P \text { and } B_{x}(p, q) \equiv \int_{0}^{x} t^{p-1}(1-t)^{q-1} d x(0<x<1) .
$$ 
that the envelope is convective, we can express the pressure, density, temperature, and coordinate at a certain position in the envelope as

$$
\begin{gathered}
P=P_{\mathrm{b}}\left(\frac{x}{x_{\mathrm{b}}}\right)^{N+1}\left(\frac{1-x}{1-x_{\mathrm{b}}}\right)^{-(N+1)}, \rho=\rho_{\mathrm{b}}\left(\frac{x}{x_{\mathrm{b}}}\right)^{N}\left(\frac{1-x}{1-x_{\mathrm{b}}}\right)^{-N}, \\
T=T_{\mathrm{b}}\left(\frac{x}{x_{\mathrm{b}}}\right)^{(N+1) /(n+1)}\left(\frac{1-x}{1-x_{\mathrm{b}}}\right)^{-(N+1) /(n+1)}, \text { and } r=R_{\mathrm{WD}} \frac{1-x}{1-x_{\mathrm{b}}} .
\end{gathered}
$$

We assume here that $n$ is also adiabatic and constant ${ }^{3}$.

A shell flash is calculated by solving the equation of the energy conservation,

$$
\frac{d s}{d T}=\left\langle\frac{\varepsilon^{(\mathrm{N})}}{T}\right\rangle,
$$

where $s$ denotes the specific entropy, and $\varepsilon^{(\mathrm{N})}$ is the specific energy generation rate by nucleosynthesis. The angle brackets in the right-hand side of the above equation denote an average over the envelope. For simplicity, we treat the envelope as one zone. The nucleosynthesis network includes 280 nuclides from $\mathrm{n}$ and $\mathrm{p}$ up to ${ }^{79} \mathrm{Br}$.

\section{3. preliminary results}

The model parameters are $M_{\mathrm{WD}} / M_{\odot}=1.2,1.25,1.3,1.35,1.38$ and $\log \left(M_{\mathrm{env}} / M_{\odot}\right)=-2.5$, $-3,-3.5,-4,-4.5,-5$. These values include the parameter range that triggers a shell flash on a helium-accreting WD [Q].

Figure $\mathbb{W}$ shows the progress and nucleosynthesis of the shell flash for the model with $M_{\mathrm{WD}}=$ $1.35 M_{\odot}$ and $\log \left(M_{\text {env }} / M_{\odot}\right)=-3.5$. The progress of the shell flash is described as follows.

1. At first, the envelope is degenerate. The temperature increases while the pressure remains almost constant.

2. The envelope gradually expands. The pressure and density decrease, and the He in the envelope is burned into $\mathrm{C}$.

3. Then, around the peak temperature, an explosive nucleosynthesis occurs. Most of $\mathrm{He}$ and $\mathrm{C}$ are burnt into heavier elements, such as $\mathrm{O}, \mathrm{Ne}$, and $\mathrm{Mg}$.

4. After the temperature maximum, the envelope begins to cool down. The temperature and density decrease.

Figure $\square$ shows several results of our calculations. A model with larger $M_{\mathrm{WD}}$ and/or $M_{\mathrm{env}}$ shows a stronger He shell flash, so that its peak temperature becomes higher. For such models that the peak temperature becomes around $10^{9} \mathrm{~K}$, a large amount of $\mathrm{Si}$ and $\mathrm{S}$ is synthesized. This result suggests that the outer layer of the progenitor WD could have a certain amount of the elements heavier than $\mathrm{C}$ and $\mathrm{O}$, which might result in the HVFs seen in the early-time spectra of SNe Ia. 

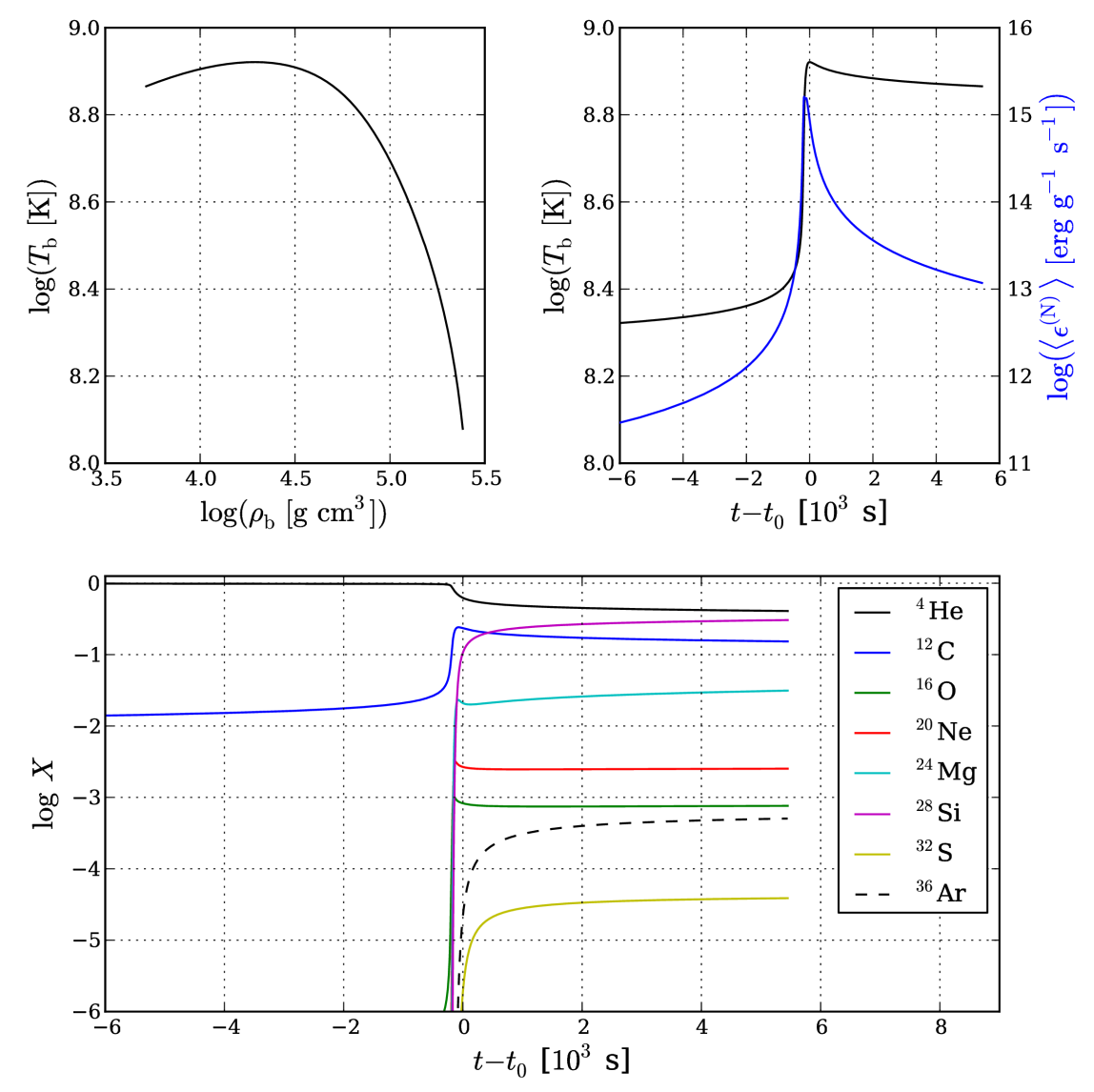

Figure 1: Progress and nucleosynthesis of the He shell flash for the model with $M_{\mathrm{WD}}=1.35 M_{\odot}$ and $\log \left(M_{\text {env }} / M_{\odot}\right)=-3.5$. Top-left: $\rho_{\mathrm{b}}-T_{\mathrm{b}}$ diagram. Top-right: time evolutions of $T_{\mathrm{b}}$ and $\left\langle\varepsilon^{(\mathrm{N})}\right\rangle$. Bottom: time evolution of the abundance around the peak temperature $\left(t=t_{0}\right)$.

However, since the peak temperature do not greatly exceed $10^{9} \mathrm{~K}$, much heavier elements (e.g., beyond $\mathrm{Ca}$ ) are not significantly produced.

As is seen in Figure 目, there remains a certain amount of He after the peak temperature (10$70 \%$ in mass fraction). Since the current calculation stops when the envelope becomes cool enough after the peak by technical reasons, the remaining He might be burnt into heavier elements if we continue the calculation. Assuming that the unburnt $\mathrm{He}$ is finally burnt into $\mathrm{C}$ and $\mathrm{O}$ with the same mass fraction through a stable He burning after a He shell flash, we can estimate a C-O ratio (see the bottom-right panel of Figure ( ). If all the nuclides synthesized by a He shell flash remain on the surface of a WD, and unless the binary evolution does not influence the remaining nuclides, the $\mathrm{C}-\mathrm{O}$ ratio could be different from $1: 1$, which is theoretically assumed.

It should be also mentioned that the temperature of some models becomes so high that the assumption of hydrostatic equilibrium breaks. The evolutions of these He shell flashes would be somewhat different; further nucleosythesis would occur and produce more $\mathrm{Ca}$. We should treat and

\footnotetext{
${ }^{3} n+1 \equiv d \ln P / d \ln T$.
} 

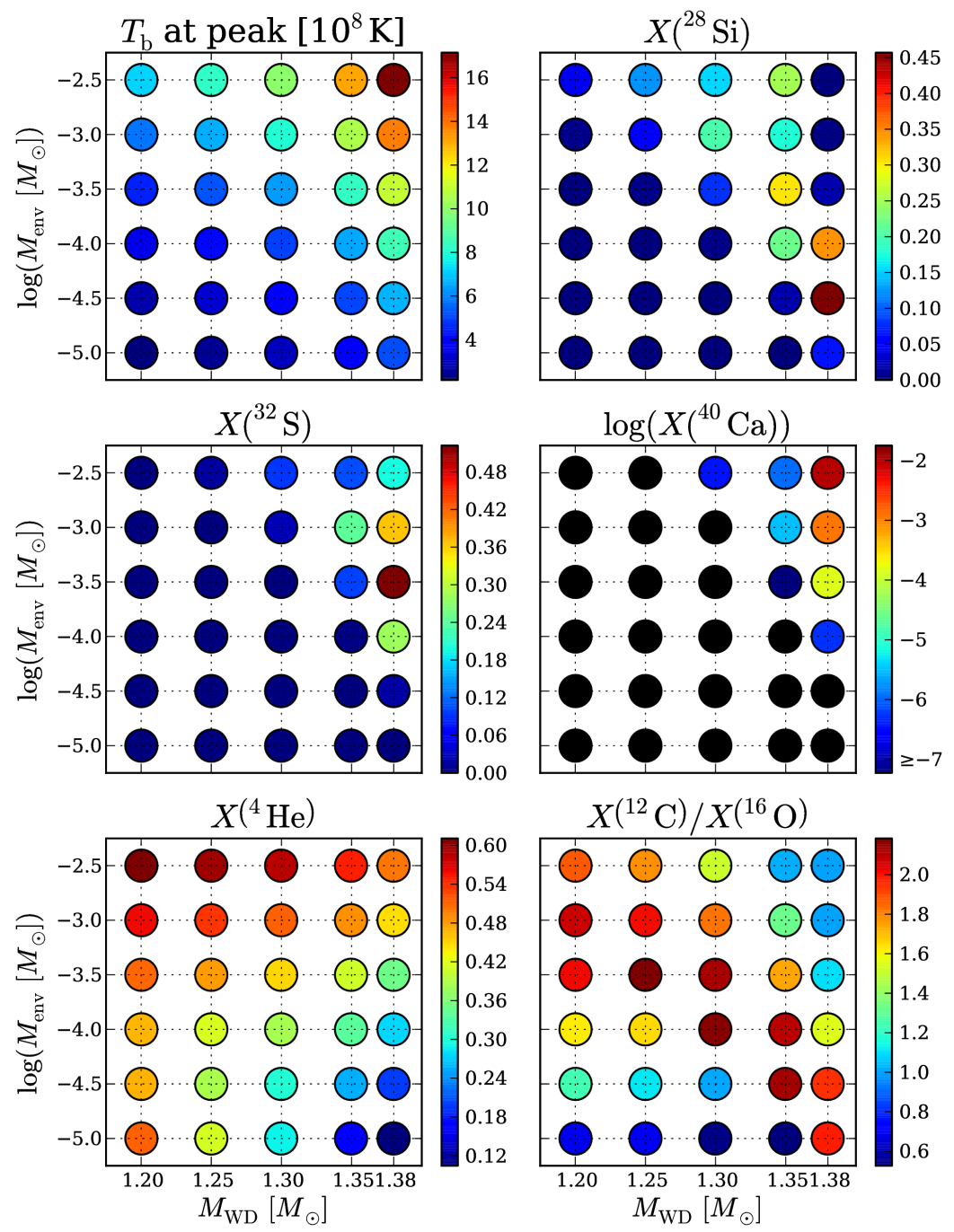

Figure 2: Summary plots of our calculation results. Top-left: peak temperature obtained during the He shell flash. Top-right, middle-left, middle-right, and bottom-left: abundance of ${ }^{28} \mathrm{~S},{ }^{32} \mathrm{Si},{ }^{40} \mathrm{Ca}$, and ${ }^{4} \mathrm{He}$ at the end of our calculations, respectively. The black circles in the middle-right panel denote that the abundance of ${ }^{40} \mathrm{Ca}$ is zero for these models. Bottom-right: estimated final $\mathrm{C}-\mathrm{O}$ ratio if the unburnt $\mathrm{He}$ is burnt into $\mathrm{C}$ and $\mathrm{O}$ with the same mass fraction. 
examine such models carefully in further studies.

\section{Summary}

We have calculated the evolutions and nucleosynthesis of the He shell flashes on the accreting WDs, by using the quasi-analytical models. Our calculations suggest that:

- A model of a heavier WD and/or with a heavier envelope shows a stronger He shell flash, and its peak temperature becomes higher.

- A He shell flash produces a significant amount of the elements (such as Si and S) which might explain the HVFs in the early-time spectra of SN Ia, while heavier elements like Ca are synthesized very little.

- Though we should treat carefully the unburnt He and models where hydrostatic equilibrium breaks, the progenitor WD seems to have other elements besides $\mathrm{C}$ and $\mathrm{O}$ in its outermost layer.

\section{Acknowledgments}

Y.K. acknowledges the Japan Society for the Promotion of Science (JSPS) for support through JSPS Research Fellowships for Young Scientists, and his work is supported by Grant-in-Aid for JSPS Fellows \#22.7641.

\section{References}

[1] W. Hillebrandt and J.C. Niemeyer, Type Ia Supernova Explosion Models, ARA\&A 38 (2000) 191-230.

[2] K. Nomoto, K, Iwamoto and N. Kishimoto, Type Ia Supernovae: Their Origin and Possible Applications in Cosmology, Science 276 (1997) 1378-1382.

[3] K. Nomoto et al., Type Ia Supernovae: Progenitors and Evolution with Redshift, in proceedings of Cosmic Explosions: Tenth Astrophysics Conference, AIP Conf. Proc. 552 (2000) 35-52 [astro-ph/0003134].

[4] D. Arnett, Supernovae and Nucleosynthesis: An Investigation of the History of Matter from the Big Bang to the Present, Princeton University Press, Princeton 1996.

[5] P.A. Mazzali et al., High-Velocity Features: A Ubiquitous Property of Type Ia Supernovae, ApJ $\mathbf{6 2 3}$ (2005) L37-L40.

[6] D. Sugimoto and M.Y. Fujimoto, A General Theory for Thermal Pulses of Finite Amplitude in Nuclear Shell- Burnings, PASJ 30 (1978) 467-482.

[7] M.Y. Fujimoto, A Theory of Hydrogen Shell Flashes on Accreting White Dwarfs. I. Their Progress and the Expansion of the Envelope, ApJ 257 (1982) 752-766.

[8] S. Wanajo, S. Hashimoto and K. Nomoto, Nucleosynthesis in ONeMg Novae: Models versus Observations to Constrain the Masses of ONeMg White Dwarfs and their Envelopes, ApJ 523 (1999) 409-431.

[9] M. Kato, I. Hachisu, S. Kiyota and H. Saio, Helium Nova on a Very Massive White Dwarf: A Revised Light-Curve Model of V445 Puppis (2000), ApJ 684 (2008) 1366-1373. 